\title{
Partial and saturation swelling in latex particles of polybutadiene, styrene-methyl methacrylate copolymers and composite particles
}

Citation for published version (APA):

Aerdts, A. M., Boei, M. M. W. A., \& German, A. L. (1993). Partial and saturation swelling in latex particles of polybutadiene, styrene-methyl methacrylate copolymers and composite particles. Polymer, 34(3), 574-579. https://doi.org/10.1016/0032-3861(93)90553-M

DOI:

10.1016/0032-3861(93)90553-M

Document status and date:

Published: 01/01/1993

Document Version:

Publisher's PDF, also known as Version of Record (includes final page, issue and volume numbers)

Please check the document version of this publication:

- A submitted manuscript is the version of the article upon submission and before peer-review. There can be important differences between the submitted version and the official published version of record. People interested in the research are advised to contact the author for the final version of the publication, or visit the $\mathrm{DOI}$ to the publisher's website.

- The final author version and the galley proof are versions of the publication after peer review.

- The final published version features the final layout of the paper including the volume, issue and page numbers.

Link to publication

\footnotetext{
General rights

- You may freely distribute the URL identifying the publication in the public portal. follow below link for the End User Agreement:

www.tue.nl/taverne

Take down policy

If you believe that this document breaches copyright please contact us at:

openaccess@tue.nl

providing details and we will investigate your claim.
}

Copyright and moral rights for the publications made accessible in the public portal are retained by the authors and/or other copyright owners and it is a condition of accessing publications that users recognise and abide by the legal requirements associated with these rights.

- Users may download and print one copy of any publication from the public portal for the purpose of private study or research.

- You may not further distribute the material or use it for any profit-making activity or commercial gain

If the publication is distributed under the terms of Article 25fa of the Dutch Copyright Act, indicated by the "Taverne" license above, please 


\title{
Partial and saturation swelling in latex particles of polybutadiene, styrene methyl methacrylate copolymers and composite particles
}

\author{
Annemieke M. Aerdts, Monique M. W. A. Boei and Anton L. German* \\ Department of Polymer Chemistry and Technology, Eindhoven University of Technology, \\ PO Box 513, $5600 \mathrm{MB}$ Eindhoven, The Netherlands \\ (Received 1 April 1992)
}

\begin{abstract}
Partial and saturation swelling experiments in latex particles of styrene methyl methacrylate copolymers, polybutadiene and composite particles containing polybutadiene with styrene and methyl methacrylate grafted were performed with the centrifugation method. Very good agreement is achieved between the experimental results of partial swelling and a simplified theoretical model. The behaviour of monomer partitioning when no monomer droplets are present is independent of polymer type, polymer crosslinking and latex particle diameter. The saturation monomer partitioning experiments also showed good agreement with a simplified thermodynamic theory. The polymer type does not influence the ratio of two monomers (styrene and methyl methacrylate) in the particle and droplet phases. Also, there is no preferential solvation of styrene in polybutadiene. This is an important result for the graft polymerization of styrene and methyl methacrylate. Differences observed in copolymer composition between graft and free copolymers thus cannot be ascribed to monomer partitioning.
\end{abstract}

(Keywords: swelling; styrene methyl methacrylate copolymers; polybutadiene; graft polymers; latex)

\section{INTRODUCTION}

Swelling behaviour of 'seed' latex particles by monomers is of great importance. For example, methyl methacrylate/ butadiene/styrene (MBS) and acrylonitrile/butadiene/ styrene (ABS) are prepared in two emulsion polymerization steps; in the first step a polybutadiene (PB) seed is prepared (the rubbery core) and in the second step a monomer mixture [e.g. styrene (S)/methyl methacrylate (MMA)] is added and copolymerized (the glassy shell). Some of the latter monomers will graft onto the PB particles. The rest of the monomer will polymerize without chemically bonding onto the PB core, the so-called 'free styrene methyl methacrylate copolymer' (SMMA-f, see Table $I$ for abbreviations used). The presence of a PB seed latex in principle may influence the behaviour of partitioning of monomers due to different interactions between monomers and polymers. Different monomer concentrations will then influence the composition of the SMMA copolymer grafted onto PB and the SMMA-f copolymer. Differences in copolymer composition between grafted SMMA (SMMA-g) and SMMA-f copolymers can affect polymer phase separation ${ }^{1}$. Moreover, the properties of graft polymers depend on the degree of grafting ${ }^{2}$, particle morphology, the amount and length of the grafts, molecular weight and in the case of copolymers, the copolymer composition and compositional distribution. The influences of process variables on the graft polymerization have been reported by several groups ${ }^{2-4}$. However, in order to understand the relations between process conditions, polymer characteristics and mechanical properties more

\footnotetext{
* To whom correspondence should be addressed
}

0032-3861/93/030574-06

(C) 1993 Butterworth-Heinemann Ltd.

574 POLYMER, 1993, Volume 34, Number 3 fundamental research is necessary. The way monomers partition between several phases has been described by several investigators ${ }^{5-9}$. The saturation swelling of latex particles has been described by Morton et al. ${ }^{5}$ and Maxwell et al. ${ }^{6}$, and the partial swelling where all monomer is solubilized in the aqueous phase and in the latex particles, by Vanzo et al. ${ }^{7}$, Gardon $^{8}$ and Maxwell et al. $^{9}$. some partitioning experiments of $\mathrm{S}$ and acrylonitrile (AN) with PB latices and films have been reported by Mathey and Guillot ${ }^{10}$. They estimated the values for the interaction parameter and the surface tension, and showed by theory that the particle size and crosslinking density of the PB latex influences the monomer concentration in PB particles. Actually the interaction parameter and surface tension are hard to determine experimentally and may also be dependent on the volume fraction of polymer $^{9}$. Experimentally ${ }^{10}$, only the $\mathrm{S}$ concentration in PB latex particles at saturation was measured. The monomer concentration of the two monomers as a function of different monomer compositions would be of interest because this gives us

Table 1 Abbreviations used for different types of polymers

\begin{tabular}{ll}
\hline PB & $\begin{array}{l}\text { Polybutadiene } \\
\text { Styrene methyl methacrylate copolymer formed } \\
\text { independently, i.e. in the absence of PB seed } \\
\text { Composite polymer of PB with grafts of S and MMA } \\
\text { PB-SMMA-g }\end{array}$ \\
SMMA-f & $\begin{array}{l}\text { Free SMMA copolymer formed during MBS graft } \\
\text { polymerization }\end{array}$ \\
MBS & $\begin{array}{l}\text { Product of grafting process, i.e. PB-SMMA-g } \\
\text { +SMMA-f }\end{array}$ \\
SMMA-g & SMMA copolymer grafted onto PB
\end{tabular}


Table 2 Seed latex recipes of PB, SMMA-i copolymers and MBS graft polymers (in parts by weight) ${ }^{a}$

\begin{tabular}{|c|c|c|c|c|c|c|}
\hline & \multirow[b]{2}{*}{ PBI } & \multirow[b]{2}{*}{ PBII } & \multicolumn{2}{|c|}{ SMMA-i } & \multirow[b]{2}{*}{ MBSI } & \multirow[b]{2}{*}{ MBSII } \\
\hline & & & $25 / 75$ & $52.5 / 47.5$ & & \\
\hline Water & 2800 & 691 & 600 & 600 & 600 & 762 \\
\hline S & - & - & 30.9 & 64.18 & 23.180 & 22.015 \\
\hline MMA & - & - & 89.1 & 55.82 & 66.820 & 62.658 \\
\hline $\mathrm{B}\left(\mathrm{PB}^{b}\right)$ & 1200 & 691 & - & - & $30^{b}$ & $29.75^{\mathrm{b}}$ \\
\hline SDS & - & - & 2.0 & 2.0 & 0.875 & - \\
\hline (S)PPS & 15.14 & 3.74 & 0.2 & 0.2 & 1.427 & - \\
\hline (S)PCB & 7.74 & 1.91 & - & - & 0.638 & - \\
\hline tDM & 12.00 & 6.91 & 1.2 & 1.2 & 0.900 & 0.850 \\
\hline CHP & - & - & - & - & - & 0.213 \\
\hline SFS & - & - & - & - & - & 0.213 \\
\hline $\mathrm{FeSO}_{4} /$ EDTA & - & - & - & - & - & 0.005 \\
\hline
\end{tabular}

${ }^{a}$ SDS, sodium dodecylsulfonate; (S)PPS, sodium- or potassium persulfate; (S) PCB, sodium- or potassium carbonate; tDM, tertiary dodecylmercaptan; CHP, cumenehydroperoxide; SFS, sodium formaldehyde sulfoxylate; $\mathrm{FeSO}_{4} /$ EDTA, complex of iron(II)sulfate and ethylenediaminetetraacetic acid

information about the monomer partitioning in copolymerization (it is known that the presence of $S$ in SAN and graft polymer influences the AN concentration in the latex particles ${ }^{11}$ ). To understand the swelling behaviour of monomers in PB, it is better to first understand the swelling behaviour of partially water soluble monomers which are good solvents for their own polymer.

In this paper, experimental results of partial and saturation swelling of the monomers $\mathrm{S}$ and MMA in SMMA copolymer formed in the absence of $P B$ seed (SMMA-i), in PB and in SMMA-f and PB-SMMA-g (from the MBS graft polymerization) are discussed and compared to predicted results from the semiempirical equations developed recently by Maxwell et $a l^{6,9}$. The following parameters were varied in the monomer partitioning experiments: latex particle diameter (of $P B$ ), crosslinking density (of PB), polymer type, copolymer composition and degree of grafting (of MBS).

\section{EXPERIMENTAL}

The PB latices were prepared by emulsifier free emulsion polymerization $^{12}$ (Table 2), leading to monodisperse seed latices. Butadiene (DSM, Geleen, The Netherlands) was purified by distillation. The polymerizations were performed in a 51 stainless steel reactor fitted with two 12-bladed turbine impellers. A conversion of $95 \%$ was reached after $20 \mathrm{~h}$. The larger PB particle diameter sizes were made in a 2.51 stainless steel reactor fitted with a helical ribbon rotator with the first $4 \mathrm{~h}$ at $80^{\circ} \mathrm{C}$ and then $92 \mathrm{~h}$ at $60^{\circ} \mathrm{C}(85 \%$ conversion $)$. The average particle sizes and particle size distributions were determined by transmission electron microscopy. The details of the PB latices are given in Table 3. Different crosslinking densities are achieved by varying the mercaptan concentrations and by polymerizing to higher final conversions ${ }^{13}$. High crosslinking densities can also be achieved by heating a $\mathrm{PB}$ latex for $8 \mathrm{~h}$ at $90^{\circ} \mathrm{C}$. The gel content was measured as an indication of the crosslinking density of the PB, and was determined by the solvent extraction method of the soluble fraction of the PB. The insoluble residue is, by definition, the gel fraction. Before using the PB for the monomer partitioning experiments, the latex was dialysed in a membrane tube in order to remove excess surfactant and monomer (Medicell International Ltd, London, UK). The water was changed five times every $4 \mathrm{~h}$ for 2 days $^{14,15}$. After this, the solid contents were determined by gravimetry.

The SMMA copolymers (SMMA-i) were made in a 11 stainless steel reactor fitted with a 12-bladed turbine impeller, under nitrogen according to a standard recipe given in Table 2. Table 2 also gives the recipe for the graft polymerization of MBS made in the same reactor. These latices were dialysed in the same manner as the PB latices. The monomers used in the monomer partitioning experiments were applied as received without any further purification. The presence of a free radical inhibitor prevents polymerization during the monomer partitioning experiments. The determination of the monomer partitioning was performed with the centrifugation method ${ }^{6,9}$. In the partial swelling experiments the latex was mixed with known amounts of MMA and in the saturation experiments with MMA and S. After $2 \mathrm{~h}$ of shaking, equilibrium was reached. It is necessary to know the solid content of the latex, the copolymer composition and for the MBS polymer also the degree of grafting. Polymer densities were calculated by the appropriate averaging of the densities of the homopolymers. For the experiments with the MBS polymers the amount of PB-SMMA-g and SMMA-f were calculated from the degree of grafting (measured by n.m.r.) ${ }^{16}$ and the total weight. The swollen polymer particles phase and aqueous phase (and monomer phase) were separated using an ultracentrifuge $\left(55000 \mathrm{rev} \mathrm{min}{ }^{-1}\right.$, Centrikon T-2060, 6-15 h) at room temperature. Monomer concentrations in polymer and aqueous phases were determined by gas chromatography. The PB and PB-SMMA-g phases were swollen in toluene with acetone as an internal standard. The SMMA-i and SMMA-f copolymers were dissolved in acetone with toluene as an internal standard. The concentration of MMA in the aqueous phase was determined after adding a standard 2-propanol solution in water to a sample of the aqueous phase. In the monomer phase samples only the monomer ratio was determined.

\section{RESULTS AND DISCUSSION}

\section{Partial swelling of latex particles by one monomer}

The partial swelling of latex particles composed of crosslinked polymer by monomer and solvents can be described by the Vanzo equation in combination with the Flory-Rehner theory ${ }^{9}$ :

$$
\begin{aligned}
& \ln \left(1-v_{\mathrm{p}}\right)+v_{\mathrm{p}}\left(1-1 / M_{\mathrm{n}}\right)+\chi v_{\mathrm{p}}^{2}+\left(2 V_{\mathrm{m}} \gamma v_{\mathrm{p}}^{1 / 3} / R_{0} R T\right) \\
& +\left(V_{\mathrm{m}} d_{\mathrm{p}} / M_{\mathrm{c}}\right)\left(v_{\mathrm{p}}^{1 / 3}-v_{\mathrm{p}} / 2\right)=\ln \left([\mathrm{M}]_{\mathrm{aq}} /[\mathrm{M}]_{\mathrm{aq}, \mathrm{sat}}\right)
\end{aligned}
$$

where $v_{\mathrm{p}}$ is the volume fraction of polymer in the latex particles, $M_{\mathrm{n}}$ is the number average degree of polymerization, $R$ is the gas constant, $T$ is the temperature, $\chi$ is

Table 3 Number average $\left(D_{n}\right)$ and weight average $\left(D_{w}\right)$ unswollen particle diameters, polydispersity $\left(P_{n}\right)$ and gel content of some PB seed latices

\begin{tabular}{lllll}
\hline & $\begin{array}{l}D_{\mathrm{n}} \\
(\mathrm{nm})\end{array}$ & $\begin{array}{l}D_{\mathrm{w}} \\
(\mathrm{nm})\end{array}$ & $P_{\mathrm{n}}$ & $\begin{array}{l}\text { Gel content } \\
(\%)\end{array}$ \\
\hline PBIa & 209.7 & 212.7 & 1.014 & 72 \\
PBIb & 209.7 & 212.7 & 1.014 & 95 \\
PBII & 305 & 401 & 1.313 & 70 \\
\hline
\end{tabular}




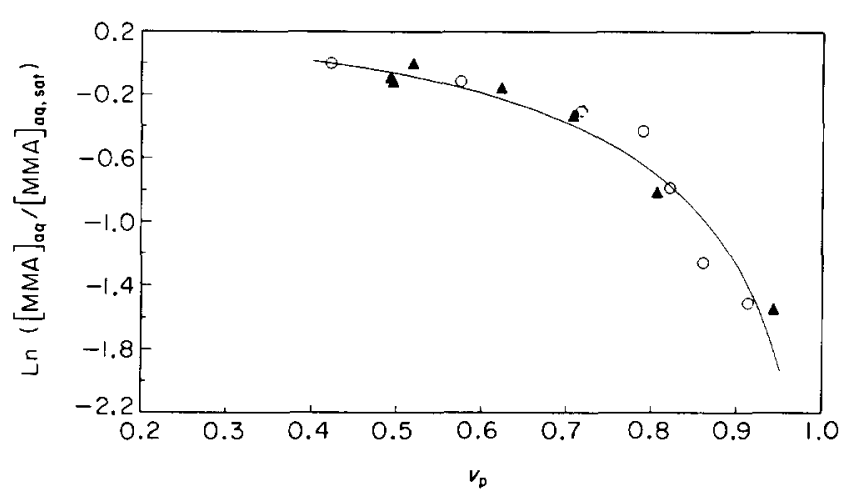

Figure 1 Comparison of theoretical predictions [according to equation (2)] with experimental results of MMA partitioning for copolymer seed latices SMMA-i (25/75) (O) and SMMA-i (50/50) (A)

the Flory-Huggins interaction parameter, $V_{m}$ is the partial molar volume of the monomer, $\gamma$ is the particlewater interfacial tension, $R_{0}$ is the unswollen radius of the latex particle, $d_{\mathrm{p}}$ is the density of the polymer, $M_{\mathrm{c}}$ is the molecular weight between crosslinks, $[\mathrm{M}]_{\mathrm{aq}}$ is the monomer concentration in the aqueous phase and $[\mathrm{M}]_{\mathrm{aq}, \mathrm{sat}}$ is the monomer concentration in the aqueous phase at saturation. The monomer partitioning behaviour between the aqueous phase and the latex particles can be predicted by this equation. For this it is necessary to know the values of $\gamma$ and $\chi$. Unfortunately, these values are hard to determine and dependent on the volume fraction of polymer. For describing the partitioning, however, it is no longer necessary to know these parameters. Maxwell et al. ${ }^{9}$ derived a semiempirical equation where the value of the sum of the residual free energy and the particle-water surface free energy terms in the Vanzo equation are estimated from the saturation swelling volume fraction of polymer ${ }^{9}$ :

$\ln \left(1-v_{\mathrm{p}}\right)+v_{\mathrm{p}}\left(1-1 / M_{\mathrm{n}}\right)+\operatorname{corr} .=\ln \left([\mathrm{M}]_{\mathrm{aq}} /[\mathrm{M}]_{\mathrm{aq}, \mathrm{sat}}\right)$

where the correction term is calculated from the Morton equation and saturation swelling data ${ }^{9}$ :

$$
\text { corr. }=-\left[\ln \left(1-v_{\mathrm{p}, \mathrm{sat}}\right)+v_{\mathrm{p}, \mathrm{sat}}\left(1-1 / M_{\mathrm{n}}\right)\right]
$$

where $v_{\mathrm{p}, \mathrm{sat}}$ is the volume fraction of polymer at saturation in the latex particles. Equation (2) showed very good agreement with experimental results for several styrene methyl acrylate (SMA) copolymers and other polymers ${ }^{9}$.

The experimental monomer concentrations of MMA in latex particles of SMMA-i, PB, SMMA-f and PBSMMA-g during partial swelling are discussed below and compared with the predictions of equation (2).

Type of polymer. In Figure 1 the partitioning results are displayed for MMA in two SMMA-i copolymers. The latices differ only in copolymer composition (see Table 2). As has already been shown for the SMA system $^{9}$, the copolymer composition has little effect on the partitioning of MA in seed latices. Although the interaction parameter will change with changing copolymer composition, the value of this interaction parameter has a small influence on the monomer partitioning. This also holds for the swelling MMA in PB latex (PBIa, Figure 2) and for PB-SMMA-g (Figure 3). From this we can conclude that for varying polymer latices, including those of PB, the free energy associated with the configurational entropy of mixing of monomer and polymer is the dominant term in equation (2). Hence, the changes in partial swelling of different seed latices with one monomer is small.

Particle size and crosslinking. The PB latices are prepared by batch emulsion polymerization processes. Depending on the conversion, temperature and mercaptan concentration, the crosslinking density will vary ${ }^{13}$. In order to show the effect of particle size and crosslinking density on the partial swelling of latex particles with MMA, experiments were performed with different PB latices (Table 3). The gel content is used as an indication of the crosslinking density. PBI has a particle diameter of $209.7 \mathrm{~nm}$, and PBIa and PBIb have gel contents of 72 and $95 \%$, respectively. PBII has a particle diameter of $305 \mathrm{~nm}$ and a gel content of $70 \%$. Experimental results of the swelling of different PBs with MMA are shown in Figure 2. Both the particle size and the crosslinking density do not significantly affect the monomer partitioning, which is in agreement with the results of Maxwell et $a l^{9}{ }^{9}$, who showed that the free energy due to the configurational entropy of mixing of monomer and polymer is the dominant factor.

Higher crosslinking densities occur when a PB seed is used for a graft polymerization, resulting from $6 \mathrm{~h}$ of polymerization at $50^{\circ} \mathrm{C}$ and from grafting. Partial swelling experiments were performed with two types of MBS (Table 4), only differing in the degree of grafting (defined as the ratio of SMMA-g over PB in PBSMMA-g). Again, no difference is found in partitioning between the PB-SMMA-g and the SMMA-f formed during graft polymerization (Figure 3). From Figures $1-3$,

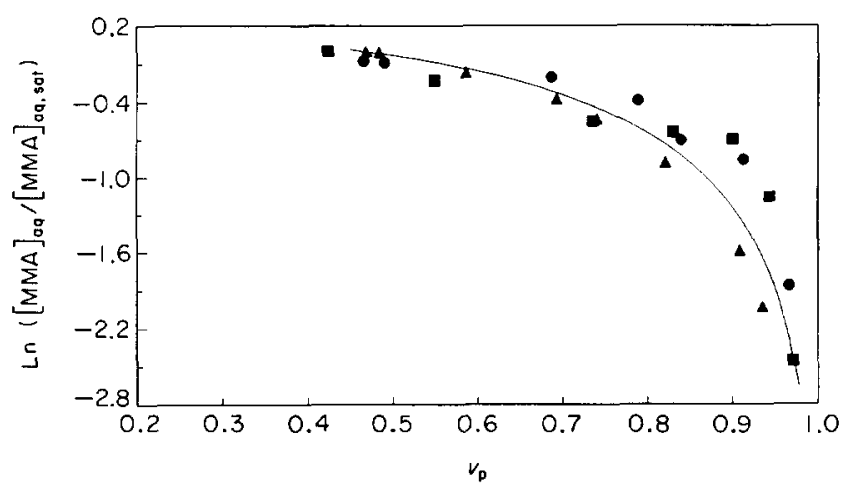

Figure 2 Comparison of theoretical predictions [according to equation (2)] with experimental results of MMA partitioning for a seed latex PBIa (O), PBIb (ם) and PBII (A)

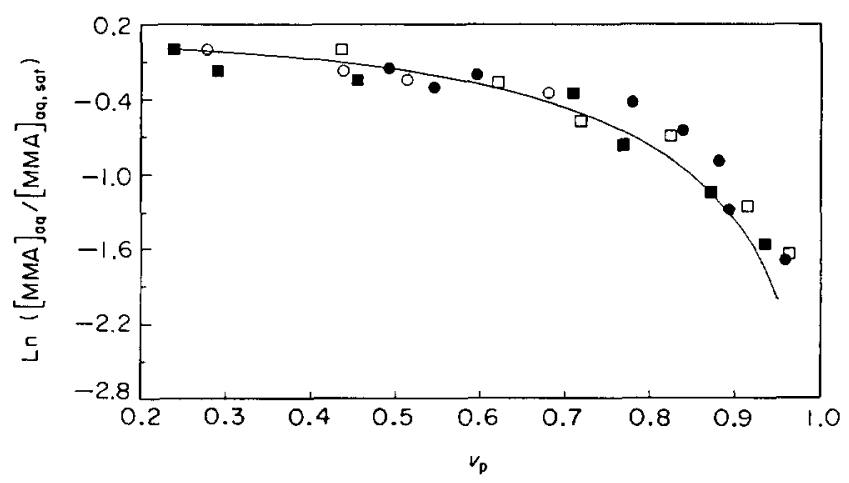

Figure 3 Comparison of theoretical predictions [according to equation (2)] with experimental results of MMA partitioning for PB-SMMA-g (O) and SMMA-f ( $\square$ ) of MBSI, and PB-SMMA-g (O) and SMMA-f $(\square)$ of MBSII 
Table 4 Characteristics of two MBS latices measured by n.m.r. ${ }^{16 a}$

\begin{tabular}{llll}
\hline & $\begin{array}{l}\text { Degree of } \\
\text { grafting }\end{array}$ & $\begin{array}{l}F_{\mathrm{s}} \text { in } \\
\text { SMMA-g }\end{array}$ & $\begin{array}{l}F_{\mathrm{s}} \text { in } \\
\text { SMMA-f }\end{array}$ \\
\hline MBSI & 0.41 & 0.18 & 0.26 \\
MBSII & 0.85 & 0.19 & 0.28 \\
\hline
\end{tabular}

${ }^{a} F_{s}$, fraction of styrene in the copolymer

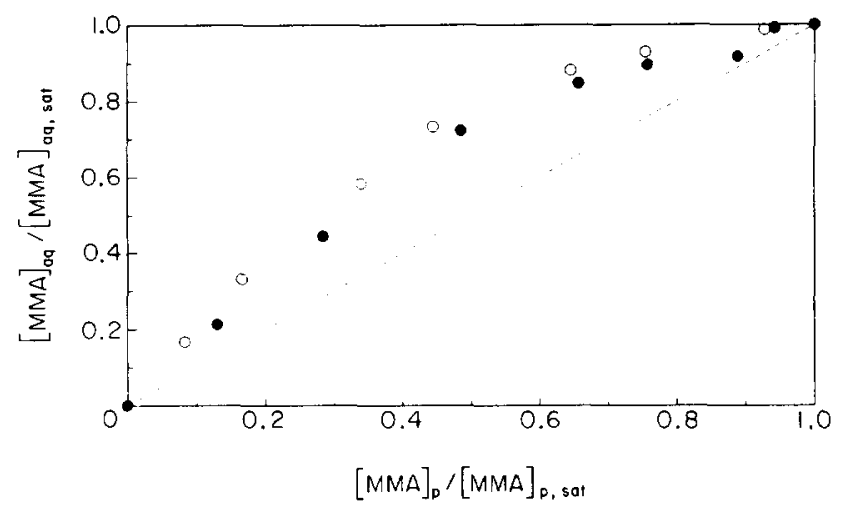

Figure 4 Experimentally determined MMA concentrations in the water phase as a function of the MMA concentration in the particle phase (concentrations normalized to saturation) in SMMA-i (25/75) (O) and in PBIa (O)

it appears that during partial swelling (stage III in emulsion polymerization) the relation between the monomer concentration in the water phase and the monomer concentration in the particle phase $\left(1-v_{p}\right)$ is convex. Thus the monomer concentration in the water phase is closer to saturation than in the particle phase. This relation does not change if the monomer concentration in the water phase is plotted on a non-logarithmic scale (Figure 4). This means that in stage III of emulsion polymerization with high water soluble monomers, a significant amount resides in the water phase, which reduces the monomer concentration and the rate of polymerization in the latex particles ${ }^{17}$.

Saturation swelling of latex particles by two monomers

At equilibrium the partial molar free energy of each monomer will be equal in each of the three phases, i.e. the polymer particle, the monomer droplet and the water phase. This leads to the following equation for monomer $i^{6}$ :

$$
\begin{gathered}
\ln v_{\mathrm{p} i}+\left(1-m_{i j}\right) v_{\mathrm{p} j}+v_{\mathrm{p}}+\chi_{i j} v_{\mathrm{p} j}^{2}+\chi_{\mathrm{ip}} v_{\mathrm{p}}^{2} \\
+v_{\mathrm{p} j} v_{\mathrm{p}}\left(\chi_{i j}+\chi_{i \mathrm{p}}-\chi_{j \mathrm{p}} m_{i j}\right)+\left(2 V_{\mathrm{m} i} \gamma v_{\mathrm{p}}^{1 / 3}\right) / R R_{0} T \\
=\ln v_{\mathrm{d} i}+\left(1-m_{i j}\right) v_{\mathrm{d} j}+\chi_{i j} v_{\mathrm{d} j}^{2}=\ln \left(\left[\mathrm{M}_{i}\right]_{\mathrm{aq}} /\left[\mathrm{M}_{i}\right]_{\mathrm{aq}, \mathrm{sat}}\right)
\end{gathered}
$$

where $v_{\mathrm{p} i}$ and $v_{\mathrm{p} j}$ are the volume fractions of monomers $i$ and $j$ in the latex particles, respectively, $\chi_{i j}$ is the interaction parameter between monomers $i$ and $j$, and $\chi_{i p}$ and $\chi_{j \mathrm{p}}$ are the interaction parameters between each of the monomers $i$ and $j$, respectively, and the polymer. The term $m_{i j}$ is the ratio of the molar volumes of monomers $i$ and $j, v_{\mathrm{d} i}$ represents the volume fraction of monomer $i$ in the droplets, and $v_{\mathrm{d} j}$ the volume fraction of monomer $j$ in the droplets, and $\left[\mathrm{M}_{i}\right]_{\mathrm{aq}}$ and $\left[\mathrm{M}_{i}\right]_{\mathrm{aq}, \text { sat }}$ are the monomer $i$ concentrations in the water phase and in the water phase at saturation, respectively.

A similar equation applies for monomer $j$.

One important assumption made in equation (4) is that a polymer seed does not change the relationship between the water and monomer phases ${ }^{6,9}$; water soluble monomers show a proportional relationship between the water phase and the monomer phase, obeying Henry's Law ${ }^{6}$. From Figure 5 it can now be derived that the same assumption of Henry's law can be made in the presence of PB seed latex particles: the concentrations of monomer in the water phase and droplets are still proportional.

Equation (4) and a similar equation for monomer $j$ can be used to predict the monomer partitioning for latex systems containing two monomers. However, the values for the interaction parameters and the interfacial tension are hard to determine and are dependent on the volume fraction of polymer in the latex particles as well as on the monomer ratio. Hence, the monomer partitioning is very hard to predict.

Simplifications to the theory of swelling of latex particles with two monomers recently made by Maxwell et al. ${ }^{6}$ are that the volume fractions of monomers are equal or similar, the interaction parameter of mixing of two monomers is rather small, and the interaction parameters for each monomer with the same polymer are equal $\left(\chi_{i \mathrm{p}}=\chi_{j \mathrm{p}}\right)$. These assumptions lead to the following result ${ }^{6}$ :

$$
\begin{aligned}
f_{\mathrm{p} i} & =f_{\mathrm{d} i} \\
f_{\mathrm{p} j} & =f_{\mathrm{d} j}
\end{aligned}
$$

where the monomer volume fraction of monomers $i$ and $j$ in the droplets is given by $f_{\mathrm{d} i(j)}$ and in the particle phases by $f_{\text {pi(j) }}$ (the volume of the polymer in the latex particles is not included in these fractions). With the additional assumption of $m_{i j}=m_{i j}$, the monomer molar fractions in the droplet and particle phases are also equal.

The actual concentrations of two monomers in the particle phase can also be estimated ${ }^{6}$. These calculations only require the values of the individual saturation concentrations of the two monomers in the latex particles. For a particular seed latex the concentration of monomer $i$ within the particles $\left(C_{i}\right)$ as a function of the fraction of monomer $i$ in the droplets, is given by ${ }^{6}$ :

$$
C_{i}=f_{\mathrm{d} i}\left[\left(C_{i \mathrm{~m}}-C_{j \mathrm{~m}}\right) f_{\mathrm{di}}+C_{j \mathrm{~m}}\right]
$$

Similarly for monomer $j$ :

$$
C_{j}=f_{\mathrm{d} j}\left[\left(C_{j \mathrm{~m}}-C_{i \mathrm{~m}}\right) f_{\mathrm{d} j}+C_{i \mathrm{~m}}\right]
$$

where $C_{i \mathrm{~m}}$ and $C_{j \mathrm{~m}}$ are the maximum saturation concentrations of monomers $i$ and $j$ in the latex particles. For the monomers S-MA, S-BA (butyl acrylate) and MA-BA in their homopolymers and copolymers good agreement was observed between experimental results and this

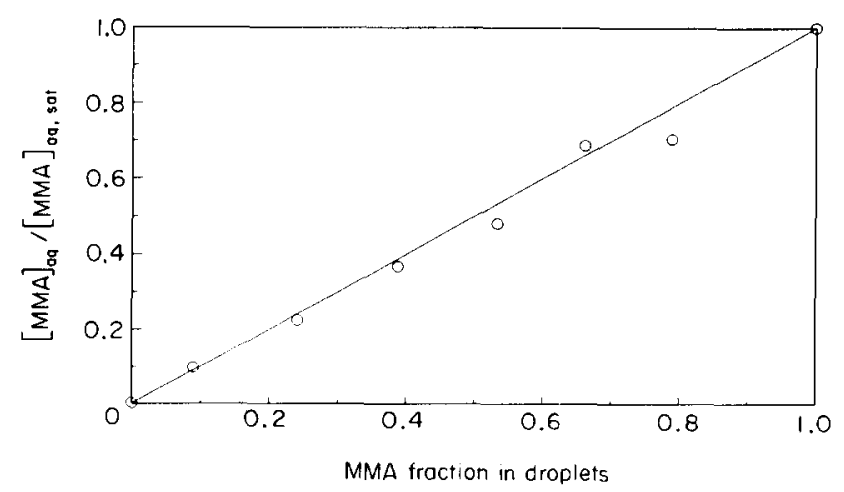

Figure 5 Experimentally determined MMA concentrations in the aqueous phase as a function of the fraction MMA in the droplet phase with $\mathrm{S}$ as comonomer in the presence of a PB latex 


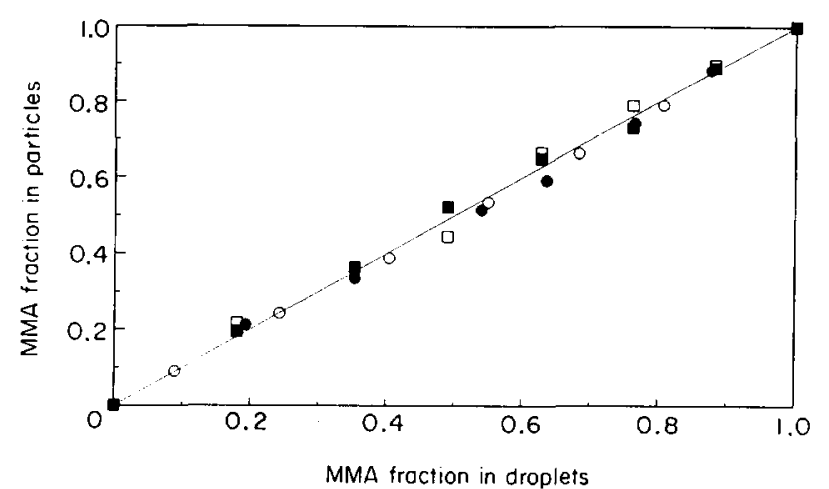

Figure 6 Experimentally determined fractions MMA in latex particles as a function of the fraction MMA in the droplet phase. MMA and S in: PBIa (O), SMMA-i (25/75) (O), SMMA-f from MBSI ( $\square$ ) and PB-SMMA-g of MBSI ( $\square$ ). The solid line gives the theoretical prediction according to equation (5).

simplified theory ${ }^{6}$. From these results it was concluded ${ }^{6}$ that the entropy of mixing of the two monomers is the dominant thermodynamic contribution, and the presence of the polymer has no significant effect upon the ratio of the two monomers in the polymer and monomer droplet phases, respectively.

Figure 6 shows the experimental results of the partitioning of $S$ and MMA between particle and monomer droplet phases in two SMMA-i copolymers of different compositions. For both polymers it can be seen that the fraction of the monomer in the droplet and particle phases are equal, as predicted by equation (5). Utilizing a different polymer, $P B$, the same characteristics are observed. This means that the assumptions made in the derivation of equation (5) are also valid for PB: the type of polymer has no effect on the mixing of two monomers. This is an important result because PB is often used as a seed in graft copolymerization, for instance in the preparation of MBS or ABS. Also for modelling purposes and for the understanding of the resulting MBS copolymer microstructure, the monomer partitioning of the two monomers is of great importance. For this reason saturation monomer swelling experiments were also performed with MBS polymers. Centrifugation of MBS polymers at saturation swelling gave four layers in the centrifugation tube. From the bottom to the top the successive layers contain: the SMMA-f copolymer, the water phase, the composite polymer PB-SMMA-g and the monomer phase. Note that the particle phase in this case is composed of individual PB-SMMA-g polymer and SMMA-f copolymer phases. The monomer concentrations and the monomer ratios in all four layers were determined. Again, no differences were observed in the fraction of monomer in the droplet and particle phases between SMMA-f, PB-SMMA-g, SMMA-i and PB as can be seen in Figure 6. From Figure 6 we can conclude that the molar fraction of the monomers is the same in PB, SMMA-i and SMMA-f copolymer, as well as in PB-SMMA-g polymer. This again shows that the ratio of the two monomers is independent of the polymer type. The observed differences in copolymer composition between PB-SMMA-g and SMMA-f (Table 4) thus cannot be ascribed to monomer partitioning effects.

In Figure $7 a$ the concentrations of the monomers $\mathrm{S}$ and MMA in the SMMA-i and SMMA-f copolymer particle phases are plotted against the fraction of MMA in the droplet phase. In Figure $7 b$ the concentrations of the monomers S and MMA in the PB and PB-SMMA-g particle phases are plotted against the fraction of MMA in the droplet phase. The absolute value of the degree of swelling depends upon polymer and monomer type (an effect of different interaction parameters and interfacial tensions). The monomer concentrations in SMMA copolymers and in the free copolymer formed during the grafting process are the same (see Figure 7a). Obviously (Figure $7 b$ ) the MMA concentration in the PB-SMMA-g polymer is higher than in $\mathrm{PB}$, and the $\mathrm{S}$ concentration is also increased, but not to the same extent. However, it should be emphasized that the higher concentrations in the particle phases do not change the ratio between the two monomers, but only could have an effect on the polymerization rates in the latex particles. The lines in Figures $7 a$ and $b$ are the predictions of equation (6); only the concentrations of the two monomers, when exclusively swelling a particular latex, are required. In these calculations very good agreement is achieved between the experimental results and the theoretically predicted monomer concentrations according to equation (6), for any ratio of monomers in the droplets.

\section{CONCLUSIONS}

MMA monomer concentrations in the latex particle, monomer droplet and water phases were measured with the centrifugation method. Experiments showed that the partitioning of MMA is independent of the type of polymer/SMMA copolymers of different composition and PB. Moreover, the partitioning of MMA in PB is independent of particle size, polymer crosslinking density
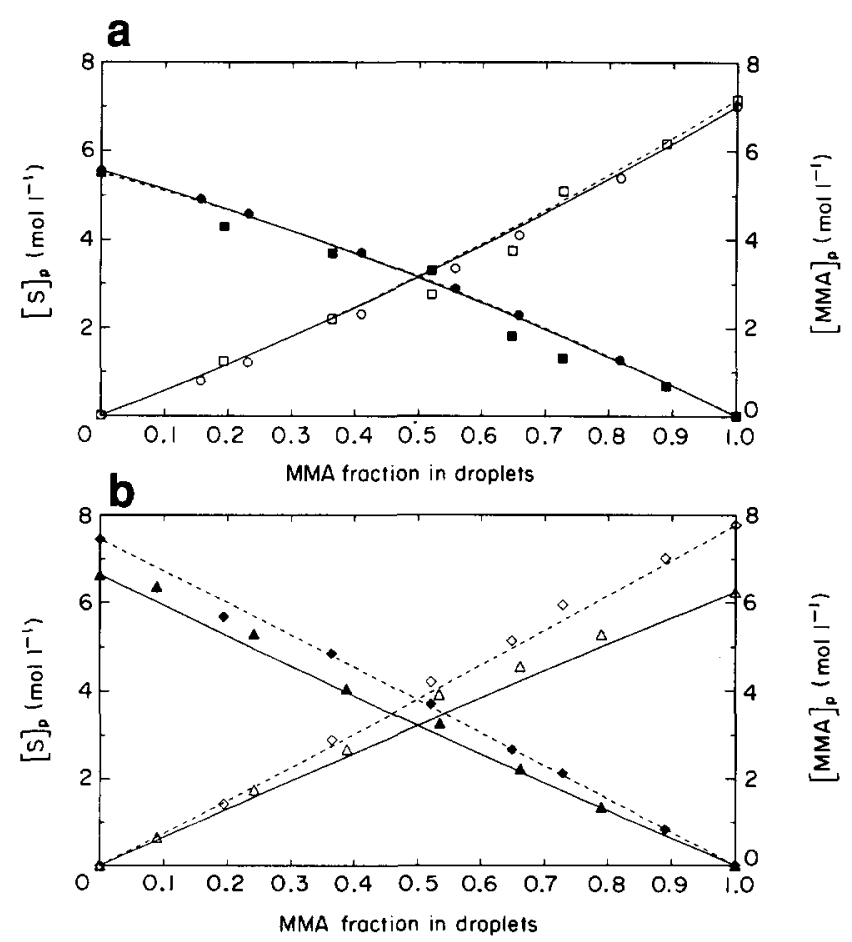

Figure 7 Experimentally determined monomer concentrations in the latex particle phase as a function of the fraction MMA in the droplet phase (symbols) compared with theoretical predictions of equation (6) (lines): (a) MMA concentrations (open symbols), $\mathrm{S}$ concentrations (closed symbols) in SMMA (25/75) copolymer (solid lines and circles) and in SMMA-f of MBSI (broken lines and squares); (b) MMA concentrations (open symbols), $\mathbf{S}$ concentrations (closed symbols) in PBIa (solid lines and triangles) and in PB-SMMA-g of MBSI (broken lines and diamonds) 
and the presence of SMMA-g. These experiments also show that the partitioning of monomer between the aqueous and latex particles phases, when there is no separate monomer phase present can be predicted using the semiempirical equation of Maxwell et al. ${ }^{9}$, which only requires the values of the saturation concentration of the monomer in the particle and aqueous phases. This result can be understood ${ }^{9}$ when realizing that the contribution of the configurational entropy of mixing of monomer and polymer dominates the free energy of monomer partitioning, and that both the interfacial free energy and residual free energy terms do not significantly influence the degree of swelling.

Experiments were also carried out at saturation swelling of different polymers by two monomers. The results of these experiments show that the mixing of the two monomers, S and MMA, is independent of the polymer type and that again the ratio of monomers in droplet and particle phases is equal. The saturation partitioning of monomers between latex particles and droplet phases could be predicted by the simplified equations of Maxwell ${ }^{6}$. This equation only requires the individual saturation concentrations of the two separate monomers in the latex particles. These results were based on the finding that the entropy of mixing of two monomers is the dominant contribution to the thermodynamic equilibrium reached ${ }^{6}$. Hence, the monomer ratio becomes independent of the type of polymer phase considered. Only the absolute value of the degree of swelling depends upon the monomer and type of polymer. This is an important result with respect to the graft polymerization of $S$ and MMA onto $P B$. In all the systems considered, namely SMMA-i, PB, PB-SMMA-g, SMMA-f and monomer droplets, the ratio of the two monomers is equal.

\section{ACKNOWLEDGEMENTS}

The authors are indebted to General Electric Plastics SA (France) for financially supporting this investigation. They also would like to thank Jenci Kurja and Ian Maxwell for their useful discussions.

\section{REFERENCES}

Molau, G. E. J. Polym. Sci. 1965, A3, 4235

2 Baum, B., Holley, W. H., Stiskin, H., White, R. A., Willis, P. B. and Wilde, A. F. Adv. Chem. Ser. 1976, 154, 263

3 Locatelli, J. L. and Riess, G. Angew. Makromol. Chem. 1973 , 28, 161

4 Locatelli, J. L. and Riess, G. Angew. Makromol. Chem. 1974, 35, 47

5 Morton, M., Kaizerman, S. and Atlier, M. W. J. Colloid Sci. $1954,9,300$

6 Maxwell, I. A., Kurja, J., Van Doremaele, G. H. J. and German, A. L. Makromol. Chem. 1992, 193, 2065

7 Vanzo, E., Marchessault, R. H. and Stannett, V. J. Colloid Sci. $1965,20,62$

8 Gardon, J. L. J. Polym. Sci. A1 1968, 6, 2859

9 Maxwell, I. A., Kurja, J., Van Doremaele, G. H. J., German, A. L. and Morrison, B. R. Makromol. Chem. 1992, 193, 2049

10 Mathey, P. and Guillot, J. Polymer 1991, 32, 934

11 Locatelli, J. L. and Riess, G. J. Polym. Sci., Polym. Lett. Edn $1973,11,257$

12 Verdurmen, E. M. F. J. and German, A. L. to be published

13 Weerts, P. PhD Thesis Technische Universiteit Eindhoven, 1990

14 Vanderhoff, J. W., Van der Hul, H. J., Tausk, R. J. M. and Overbeek, J. TH.G. in 'Clean Surfaces: their Application and Characterization for Interfacial Studies' (Ed. G. Goldfinger), Marcel Dekker Inc., New York, 1990

15 Ahmed, S. M., El Aasser, M. S., Pauli, G. H., Poehlein, G. W. and Vanderhoff, J. W. J. Colloid Interf. Sci. 1980, 2, 73

16 Aerdts, A. M. and German, A. L. to be published

17 Maxwell, I. A., Verdurmen, E. M. F. J. and German, A. L. Makromol. Chem. in press 